\title{
Executive control deficits and lesion correlates in acute left hemisphere stroke survivors with and without aphasia
}

\author{
Erin L. Meier ${ }^{1}$ - Catherine R. Kelly ${ }^{2}$ Emily B. Goldberg ${ }^{3}$ - Argye E. Hillis El, $^{2,5}$ \\ Accepted: 1 October 2021 / Published online: 14 October 2021 \\ (c) The Author(s), under exclusive licence to Springer Science+Business Media, LLC, part of Springer Nature 2021
}

\begin{abstract}
In contrast to the traditional definition of the disorder, many individuals with aphasia exhibit non-linguistic cognitive impairments, including executive control deficits. Classic lesion studies cite frontal lobe damage in executive dysfunction, but more recent lesion symptom-mapping studies in chronic aphasia present mixed results. In this study, we compared executive control abilities of acute stroke survivors with and without aphasia and investigated lesion correlates of linguistic and non-linguistic cognitive tasks. Twenty-nine participants with acute left hemisphere stroke resulting in aphasia $(\mathrm{n}=14)$ or no aphasia $(n=15)$ completed clinical MRI and testing, including three NIH Toolbox Cognition Batteries (Pattern Comparison Processing Speed, Flanker Inhibitory Control and Attention, and Dimensional Change Card Sort Tests) and the Boston Naming Test. We compared performance between groups using Wilcoxon rank sum tests. We used Least Absolute Shrinkage and Selection Operator Regression to identify neural markers (percent regional damage, hypoperfusion within vascular territories, and total lesion volume) of executive control deficits and anomia. Group performance was comparable on the Pattern Comparison Processing Speed Test, but people with aphasia had poorer standard scores, lower accuracy, and slower response times on the Dimensional Change Card Sort Test than people without aphasia. Damage to extrasylvian regions (dorsolateral prefrontal cortex, intraparietal sulcus) was related to executive control deficits, whereas language network damage (to inferior frontal and superior and posterior middle temporal gyri) was linked to naming impairments. These results suggest people with aphasia can exhibit comorbid executive control impairments linked to damage outside classic language network areas.
\end{abstract}

Keywords Acute stroke $\cdot$ Aphasia $\cdot$ Cognition $\cdot$ Executive control $\cdot$ Lesion

\section{Introduction}

Approximately $30 \%$ of acute stroke survivors present with aphasia (Engelter et al., 2006; Flowers et al., 2016), a disorder classically defined by receptive and/or expressive language

Erin L. Meier

e.meier@northeastern.edu

1 Department of Communication Sciences and Disorders, Northeastern University, 360 Huntington Avenue, 228C FR, Boston, MA 02215, USA

2 Department of Neurology, Johns Hopkins School of Medicine, Baltimore, MD, USA

3 Department of Communication Science and Disorders, University of Pittsburgh, Pittsburgh, PA, USA

4 Department of Cognitive Science, Johns Hopkins University, Baltimore, MD, USA

5 Department of Physical Medicine and Rehabilitation, Johns Hopkins School of Medicine, Baltimore, MD, USA deficits with spared non-linguistic cognition. In contrast to this definition, many people with aphasia (PWA) exhibit varied cognitive deficits (Fonseca et al., 2017 for review), including impaired executive control, an umbrella term that comprises many narrower constructs (e.g., attention, inhibition, working memory, updating, switching) (Miyake et al., 2000b). Executive dysfunction is common in acute stroke (e.g., Nys et al., 2005; Zinn et al., 2007), and cognitive deficits within the first three months post-stroke-including executive dysfunction-predict future recovery in stroke in general (Nys et al., 2005; Park et al., 2015) and PWA specifically (El Hachioui et al., 2014). In chronic aphasia, higher pre-treatment executive control predicts better language therapy outcomes (Simic et al., 2019). Despite its relevance to recovery, executive dysfunction in acute stroke survivors with versus without aphasia is underspecified.

Historically, frontal lobe syndrome is synonymous with executive dysfunction (Keil \& Kaszniak, 2002). Some recent lesion-symptom mapping (LSM) studies in chronic aphasia have implicated dorsolateral prefrontal cortex damage (DLPFC) in 
executive dysfunction (Alyahya et al., 2020; Lacey et al., 2017), whereas other studies have reported different or additional regions (e.g., left middle and superior temporal and fusiform gyri, inferior parietal lobe, temporooccipital cortex, occipital and frontal poles) (Baldo et al., 2005, 2010; Schumacher et al., 2019) or no significant lesion correlates (Butler et al., 2014; Halai et al., 2017, 2018). Possible reasons for discrepant findings may be differences in testing batteries between studies and/or variance in reorganization of brain-behavior relationships by the chronic post-stroke recovery stage (Hartwigsen \& Saur, 2019; Hillis, 2007; Kiran, 2012), resulting in a misalignment of chronic lesion correlates of executive dysfunction across investigations. LSM of multiple measures in acute stroke lessens these issues.

Therefore, in this study, we aimed to 1) characterize executive control deficits in acute stroke survivors with and without aphasia and 2) distinguish lesion correlates of non-linguistic and linguistic deficits. We hypothesized that both groups would exhibit executive control deficits but that deficits would be more pronounced in PWA compared to participants without aphasia (PWoA) based on prior subacute and chronic stroke studies (Baldo et al., 2010; Bonini \& Radanovic, 2015; Lee \& Pyun, 2014; Spaccavento et al., 2019; Yao et al., 2020). Intrinsic networks that mediate different higher-level thinking skills are spatially dissociable (Fedorenko \& Thompson-Schill, 2014). Thus, we predicted damage to cognitive network regions would be associated primarily with executive control impairments, whereas damage to language network regions would be associated primarily with language (e.g. naming) impairments.

\section{Methods}

Thirty-one patients admitted to Johns Hopkins Hospital with an acute left hemisphere (LH) stroke between February 2019 and November $2020^{1}$ were recruited as part of a larger stroke recovery project. Inclusionary criteria included normal/corrected-tonormal vision and hearing, premorbid proficiency in English, and ability to complete testing protocols. The exclusionary criterion was history of a neurological condition affecting the brain other than acute stroke or prior lacunar-only infarct $(n=10)$. The final sample included 29 patients ( 13 women; mean age: $60.76 \pm 12.65$ years). Different data from some participants were included in recent publications (Goldberg et al., 2021; Keator et al., 2020; Keser et al., 2020, 2021; Meier et al., 2020).

\section{Behavioral assessment}

We assessed non-linguistic cognition using three NIH Toolbox Cognition Batteries (Gershon et al., 2013), selected for

\footnotetext{
${ }^{1}$ Note that acute stroke participant recruitment and data collection were paused from mid-March through late September 2020 due to the COVID-19 pandemic.
}

their quick administration times and validated psychometric properties in healthy adults (Heaton et al., 2014; Weintraub et al., 2014) and individuals with acquired brain injury (Tulsky et al., 2017; Weintraub et al., 2014). Participants completed the Pattern Comparison Processing Speed (PCPS) Test, an assessment of visual processing speed; the Flanker Inhibitory Control and Attention Test, an assessment of visual attention and executive control; and the Dimensional Change Card Sort (DCCS) Test, another executive function task tapping cognitive flexibility. For each test, the NIH Toolbox application outputs an age-corrected standard score, generated by comparing an individual's combined accuracy and response times to normative data. We also collated trial-by-trial accuracy and calculated standardized reaction times (zRTs) on correct trials to disentangle these effects. Supplemental Material provides additional administration and scoring details.

We administered the Western Aphasia Battery-Revised (WAB-R; Kertesz, 2007) to measure auditory comprehension and verbal expression and the 30-item version of the Boston Naming Test (BNT; Fisher et al., 1999) to measure object naming. We used the WAB-R Aphasia Quotient (AQ) to determine aphasia severity and classify participants as PWA $(\mathrm{AQ}<93.8 ; \mathrm{n}=14)$ or PWoA $(\mathrm{AQ} \geq 93.8 ; \mathrm{n}=15)$. In clinical practice, this cutoff is often used to determine presence of aphasia and need for speech-language pathology services and as such, constitutes a clinically meaningful division of participants in this study. PWA and PWoA were matched in demographics and days post-stroke onset (Table 1).

\section{Neuroimaging methods}

Upon hospital admission, patients underwent a clinical imaging protocol that included diffusion-weighted imaging, fluidattenuated inversion recovery (FLAIR), and T1-weighted sequences acquired on a Siemens $3 \mathrm{~T}(\mathrm{n}=11)$ or $1.5 \mathrm{~T}$ $(n=17)$ magnet. Imaging parameters varied between participants and are reported in full in Supplemental Tables 1-3. We manually delineated stroke lesions on clinical images, normalized each map, and obtained a total lesion volume map for each participant; more information regarding these procedures is included in Supplemental Material.

Using Matlab scripts based on MarsBaR routines (Brett et al., 2002), we intersected each participant's lesion map with LH regions of interest (ROIs) from the language network (e.g., Fridriksson et al., 2018; Price, 2012) and networks implicated in executive control, including the default mode network (DMN; Raichle et al., 2001), dorsal attention network (DAN; Fox et al., 2006), and frontoparietal network (FPN; Cole et al., 2013). Network ROIs are depicted in Fig. 1 and listed in the Fig. 1 legend; additional information is provided in Supplemental Material. For each participant, we calculated total lesion volume 
Table 1 Comparisons between participants with and without aphasia in demographics

\begin{tabular}{lllll}
\hline & $\begin{array}{l}\text { Full sample } \\
(\mathrm{n}=29)\end{array}$ & PWA $(\mathrm{n}=14)$ & $\begin{array}{l}\text { PWoA } \\
(\mathrm{n}=15)\end{array}$ & p-value \\
\hline Age (in years) & $60.76(12.65)$ & $62.29(9.80)$ & $59.33(15.05)$ & 0.631 \\
Sex ( $n$ Women:Men) & $13: 16$ & $6: 8$ & $7: 8$ & 1.00 \\
Education (in years) & $14.59(2.51)$ & $14.21(2.55)$ & $14.93(2.52)$ & 0.392 \\
Handedness ( $n$ Right:Left) & $28: 1$ & $13: 1$ & $15: 0$ & 0.483 \\
Days Post-Stroke & $5.51(3.67)$ & $5.28(3.00)$ & $5.73(4.30)$ & 0.842 \\
\hline
\end{tabular}

Means and standard deviations (M (SD)) are reported for continuous variables for the full sample, participants with aphasia (PWA), and participants without aphasia (PWoA). Wilcoxon rank sum tests were used to compare continuous variables between PWA and PWoA. Fisher's exact tests were used to compare categorical variables between groups and percent damage to each ROI and network. The lesion overlay is shown in Supplemental Fig. 1.

Important for acute stroke, we measured hypoperfusion using the NIH FLAIR Hyperintense Vessels (FHV) rating schema (Reyes et al., 2017). We rated six LH territories (anterior and posterior cerebral artery territories, middle cerebral artery [MCA] territory split by frontal, insular, temporal, and parietal lobes) on a scale from 0 (no FHVs) to $2(3+$ FHVs/slice or $3+$ slices with FHVs).

A

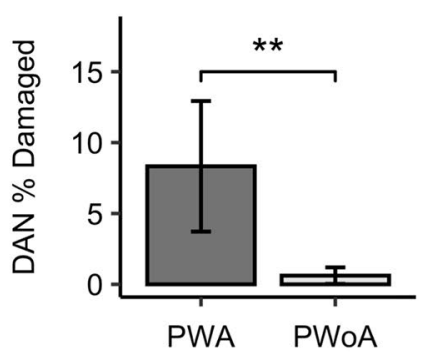

B
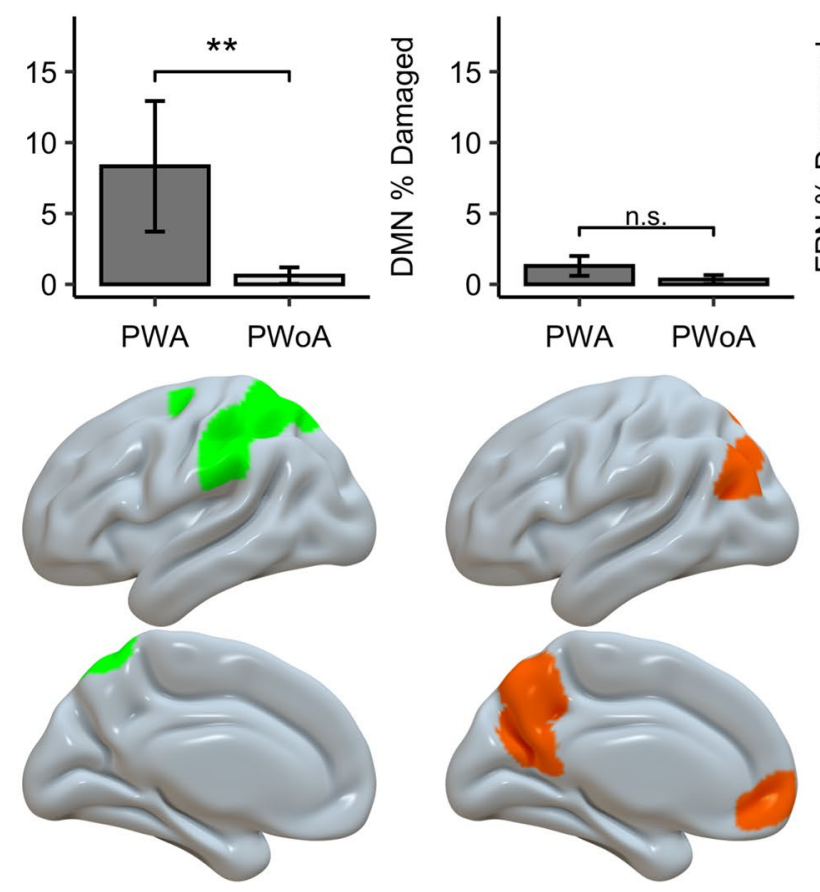

Fig. 1 Comparisons between participants with aphasia (PWA) and participants without aphasia (PWoA) in percent damaged tissue in the (A) dorsal attention network (DAN), (B) default mode network (DMN), (C) frontoparietal network (FPN), and (D) language network (LN). Comparisons were performed using FDR-corrected Wilcoxon rank sum tests. $* * q<0.01$ and not significant (n.s.). Regions of interest (ROIs) for the DAN (in neon green) included the frontal eye field and intraparietal sulcus. DMN ROIs (in orange) included the lateral

\section{Statistical analyses}

To address aim \#1, we used Wilcoxon rank sum tests to compare task performance (the three NIH Toolbox standard scores and BNT percent correct) and lesion measures (total lesion volume, total hypoperfusion, and percent damage to each network) between PWA and PWoA. We applied a Benjamini and Hochberg (1995) false discovery rate (FDR, $q=0.05$ ) correction across four task and six lesion comparisons. To

C


parietal cortex, medial prefrontal cortex, and posterior cingulate cortex. FPN ROIs (in violet) included the dorsolateral prefrontal cortex and posterior parietal cortex. LN ROIs (in cyan) included the inferior frontal gyrus (pars orbitalis, triangularis, and opercularis), the supramarginal and angular gyri, the superior and middle temporal poles, and mid and posterior portions of the superior, middle, and inferior temporal gyri 
disentangle the contributions of accuracy and response time data to the standard score comparisons, we conducted six FDR-corrected Wilcoxon tests comparing NIH Toolbox accuracy and zRTs between groups. Spearman correlations between NIH Toolbox scores and language data across the entire group are also reported in Supplemental Table 4.

To address aim \#2, we conducted LSM analyses ${ }^{2}$ using Least Absolute Shrinkage and Selection Operator (LASSO) regression, a method that imposes regularization that shrinks some parameter estimates to zero, resulting in a sparse final model with optimal prediction capacity. LASSO regression is useful when predictors exhibit high collinearity and outnumber participants (Meinshausen \& Yu, 2009), as in this study. We ran four LASSO models in which the dependent variable was the age-corrected standard score from one of the NIH Toolbox tasks or BNT percent correct; the independent predictors were ROI percent damage, hypoperfusion ratings, and total lesion volume. If fewer than $10 \%$ of the sample $(\mathrm{n}<3)$ had hypoperfusion or damage to a given region, that metric was excluded from analyses. We used the glmnet package (Friedman et al., 2010) in R to implement LASSO with standard features, utilizing a leave-oneout cross validation method to select the lambda value that was associated with the smallest mean cross-validated error per model. LASSO regressions were one-tailed due to the assumption that greater brain damage would be associated with worse, not better, performance.

\section{Results}

\section{Aim \# 1: Comparisons between PWA and PWoA}

Three participants did not complete the DCCS Test due to task difficulty or time constraints. Due to an administration error, standard scores were not obtained for one PWA. Of the remaining participants, 11 of 12 stroke survivors with aphasia had standard scores below normal limits $(<85)$ on all three NIH Cognition Batteries, whereas only two of 14 PWoA were impaired on all three tasks. Using Fisher's exact tests, comparable numbers of patients in each group had impaired PCPS Test (OR $=3.501, q=0.332$; impaired: $11 / 13$ PWA, 9/15 PWoA) and Flanker $(\mathrm{OR}=1.360, q=1.00$; impaired 11/13 PWA, 12/15 PWoA) performance, but significantly more PWA $(n=11 / 12)$ were impaired on the DCCS Test compared to PWoA $(n=2 / 14)$ $(\mathrm{OR}=49.465, q<0.001)$. Consistent with these results, standard scores did not significantly differ between groups for the PCPS (W=140.0, $q=0.068$ ) or Flanker (W=128.0, $q=0.185$ )

\footnotetext{
${ }^{2}$ Note that the term "LSM" is used in the current study to indicate the process of linking lesion characteristics and behavioral symptoms and not traditional univariate or multivariate voxel-based LSM analysis.
}

Tests, but PWA had significantly lower standard scores on the DCCS Test than PWoA (W=154.5, $q=0.003$ ) (Table 2).

Follow-up analyses compared NIH Toolbox test accuracy and zRTs between groups. Accuracy did not significantly differ between groups for the PCPS Test $(\mathrm{W}=112.0$, $q=0.738$ ), but PWA had significantly lower accuracy on the Flanker ( $\mathrm{W}=148.5, q=0.033$ ) and DCCS Test (W $=146, q=0.004$ ) than PWoA. PWA and PWoA did not significantly differ in zRTs for the PCPS Test $(\mathrm{W}=61.0$, $q=0.071$ ), but PWA had significantly slower RTs than PWoA for the Flanker (W=52.0, $q=0.033)$ and DCCS Test $(\mathrm{W}=23.0, q=0.004)$. PWA also correctly named significantly fewer items on the BNT than PWoA (W $=176.0$, $q=0.005)$.

Regarding brain measures, we found PWA had significantly greater damage to the DAN ( $\mathrm{W}=29.5, q<0.01)$ and greater total lesion volume $(\mathrm{W}=45.0, q=0.045)$ than PWoA. The other comparisons of network metrics $(q>0.119)$ and total hypoperfusion per summed FHV ratings ( $\mathrm{W}=97.0, q=0.824$ ) were not significant after multiple comparison correction. Figure 1 shows the network results.

\section{Aim \#2: Lesion correlates of anomia and executive dysfunction across the sample}

Two participants were excluded from the LSM analyses due to unusable imaging data. The LASSO model results $^{3}$ are reported in Table 3 and depicted in Fig. 2. The LASSO for PCPS Test standard scores resulted in a null model. Within the Flanker LASSO model, retained predictors included damage to the intraparietal sulcus (IPS) and parietal hypoperfusion. The model predicting DCCS scores included damage to IPS and DLPFC; hypoperfusion in parietal, frontal, and temporal lobe regions; and total lesion volume. The LASSO model for BNT naming included damage to inferior frontal gyrus, pars orbitalis (IFGorb) and pars triangularis (IFGtri), superior temporal gyrus (STG), and posterior middle temporal gyrus (pMTG) as well as hypoperfusion within the parietal and temporal lobes and posterior cerebral artery territory.

\section{Discussion}

In this study, we compared executive control in acute LH stroke survivors with and without aphasia and determined lesion correlates of executive dysfunction and anomia. PWA and PWoA did not significantly differ on all PCPS

\footnotetext{
${ }^{3}$ In LASSO regression, the combination of retained predictors yields a meaningful result outside of traditional significance testing. Nonetheless, $p$-values are reported in Table 3 to assist in interpretation of the strength of the predictors.
} 


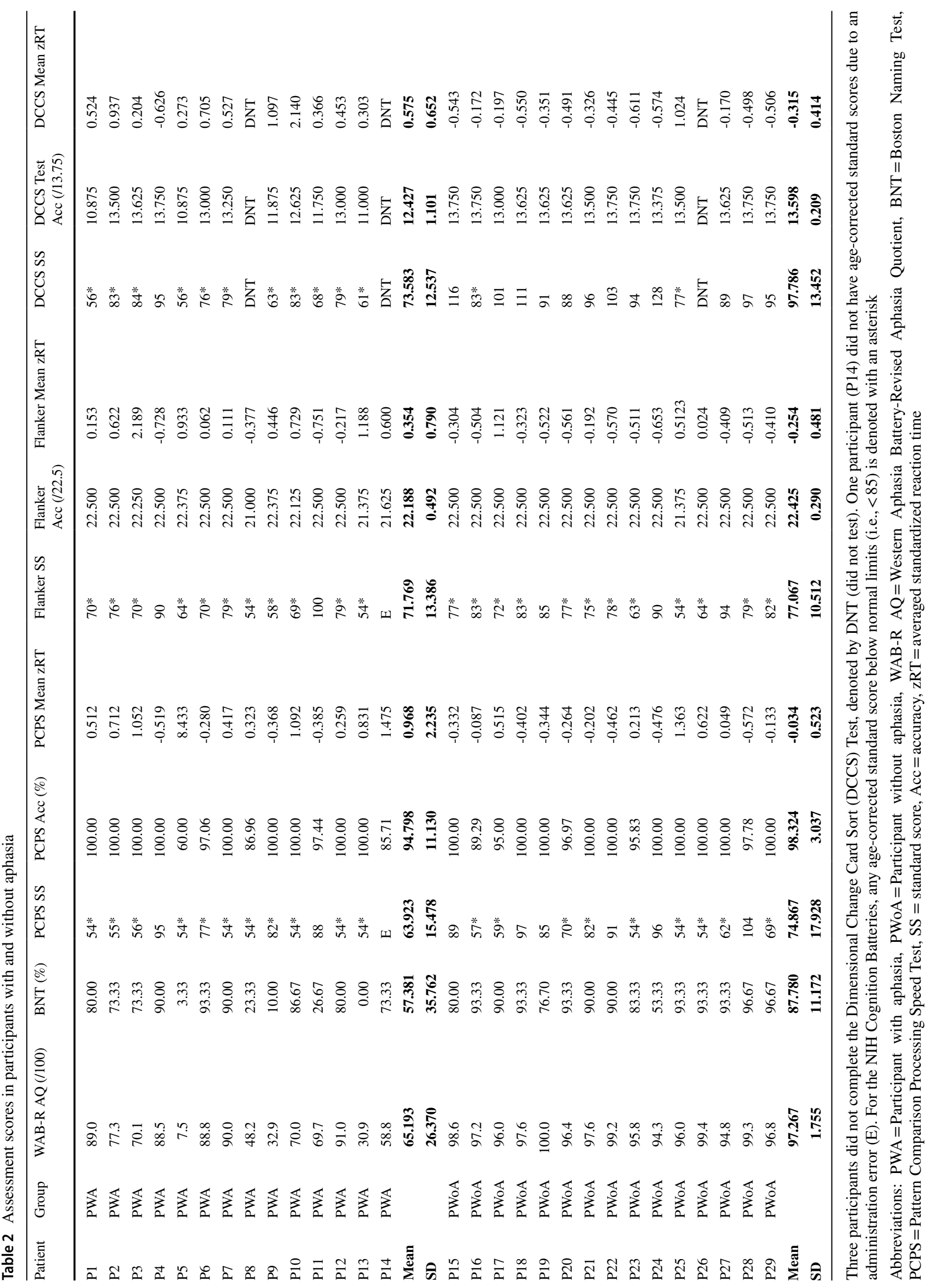


Table 3 Left hemisphere lesion correlates of performance on cognitive-linguistic measures

\begin{tabular}{|c|c|c|c|c|c|c|}
\hline \multirow[t]{2}{*}{ Predictor } & \multicolumn{2}{|c|}{ Flanker standard score } & \multicolumn{2}{|c|}{$\begin{array}{l}\text { DCCS test standard } \\
\text { score }\end{array}$} & \multicolumn{2}{|c|}{$\begin{array}{l}\text { Boston naming test } \\
\text { (percent correct) }\end{array}$} \\
\hline & Adj. Coef & $p$ & Adj. Coef & $p$ & Adj. Coef & $p$ \\
\hline \% Damage IPS (DAN) & -0.114 & 0.009 & -0.204 & 0.007 & - & - \\
\hline \% Damage DLPFC (FPN) & - & - & -0.118 & 0.078 & - & - \\
\hline \% Damage IFGorb (Language) & - & - & - & - & -0.038 & 0.177 \\
\hline \% Damage IFGtri (Language) & - & - & - & - & -0.494 & $\mathbf{0 . 0 1 0}$ \\
\hline \% Damage STG (Language) & - & - & - & - & -0.315 & 0.052 \\
\hline \% Damage pMTG (Language) & - & - & - & - & -0.201 & $\mathbf{0 . 0 4 8}$ \\
\hline FHV MCA Frontal & - & - & -0.006 & 0.386 & - & - \\
\hline FHV MCA Parietal & -0.172 & 0.002 & -0.292 & 0.026 & -0.519 & $\mathbf{0 . 0 1 0}$ \\
\hline FHV MCA Temporal & - & - & -0.031 & 0.203 & -0.120 & 0.205 \\
\hline FHV PCA & - & - & - & - & -0.021 & 0.453 \\
\hline Total Lesion Volume & - & - & -0.144 & 0.020 & - & - \\
\hline
\end{tabular}

Double dashes indicate the variable was not retained in the model. Bolded text indicates significant predictors at $p<0.05$. The LASSO regression for the Pattern Comparison Processing Speed (PCPS) Test resulted in a null model and is not shown. The network to which each region belongs is shown in parentheses in the first column. Note that the following variables were excluded from analysis due to damage/hypoperfusion in $<10 \%$ of the sample: medial prefrontal cortex, superior and middle temporal poles, inferior temporal, inferior posterior temporal, and middle temporal gyri, and anterior cerebral artery FHV ratings

Abbreviations: Coef. $=$ adjusted coefficient, $\mathrm{DAN}=$ dorsal attention network, $\mathrm{DCCS}=$ Dimensional Change Card Sort, DLPFC $=$ dorsolateral prefrontal cortex, FHV = fluid attenuated inversion recovery (FLAIR) hyperintense vessel ratings, FPN $=$ frontoparietal network, IFGorb $=$ inferior frontal gyrus, pars orbitalis, IFGtri $=$ inferior frontal gyrus, pars triangularis, IPS $=$ intraparietal sulcus, MCA = middle cerebral artery territory, $\mathrm{PCA}=$ posterior cerebral artery, $\mathrm{pMTG}=$ posterior middle temporal gyrus, $\mathrm{STG}=$ superior temporal gyrus
Test measures and Flanker standard scores, whereas PWA had significantly poorer overall performance, lower accuracy, and slower response times on the DCCS Test compared to PWoA. Regional lesion correlates of executive dysfunction and anomia differed, but hypoperfusion (particularly within the parietal lobe) predicted performance across tasks.
On average, accuracy was high for both groups on the PCPS Test, yet $71 \%$ of patients demonstrated impaired performance per standard scores. Given that standard scores reflect combined accuracy and reaction time information, these results suggest that the ability to accurately execute basic non-linguistic visual attention tasks may be intact in many patients following LH stroke, including PWA
Fig. 2 Region of interest (ROI) damaged tissue metrics retained in the LASSO regressions for the Flanker Inhibitory Control and Attention Test, Dimensional Change Card Sort (DCCS) Test, and Boston Naming Test (BNT). ROI hues reflect the model(s) in which the ROI was included according to the color key. Retained hypoperfusion ratings and total lesion volume are not depicted. DLPFC $=$ dorsolateral prefrontal cortex, IFGorb = inferior frontal gyrus, pars orbitalis, IFGtri $=$ IFG, pars triangularis, IPS = intraparietal sulcus, pMTG $=$ posterior middle temporal gyrus, STG = superior temporal gyrus

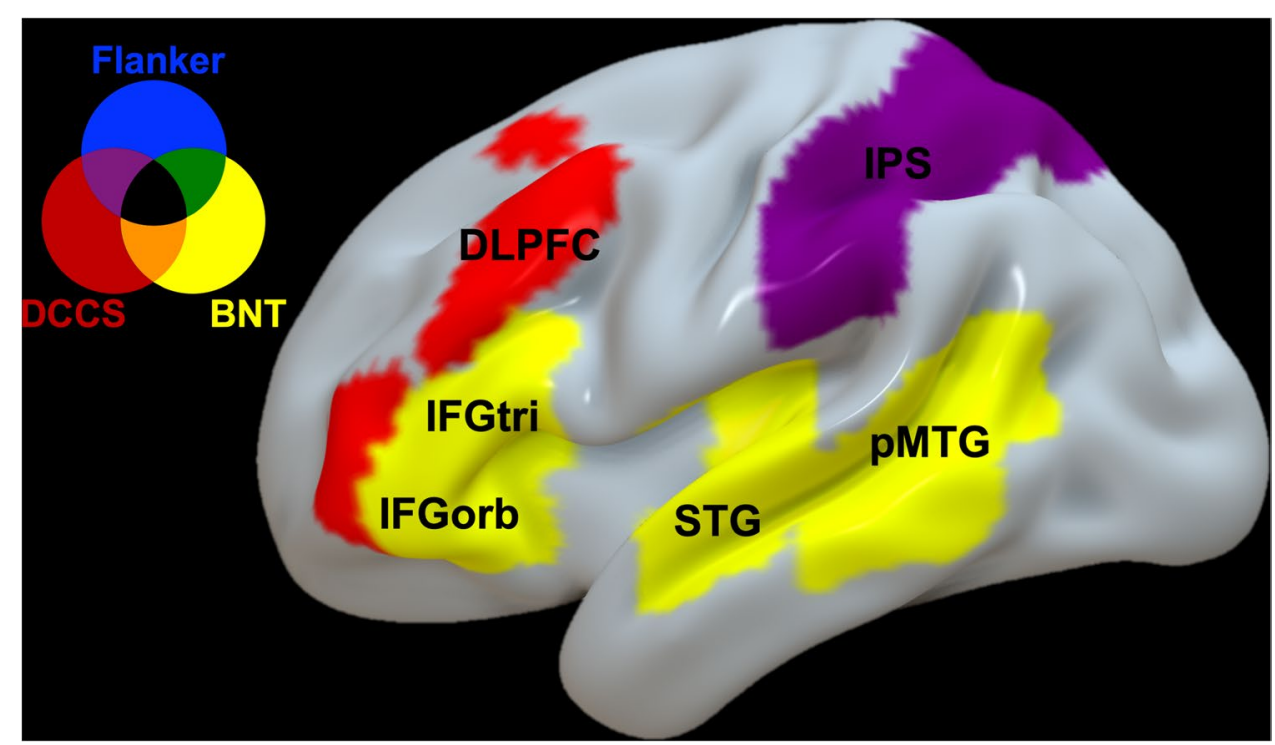


(El Hachioui et al., 2014; Kuzmina \& Weekes, 2017), yet patients may require extra response time beyond what is required for healthy age-matched peers (Faroqi-Shah \& Gehman, 2021; Korda \& Douglas, 1997; Kuzmina \& Weekes, 2017; Villard \& Kiran, 2015, 2018; c.f. Lee et al., 2020). Interestingly, Faroqi-Shah and Gehman (2021) found that linguistic task processing speed differences between individuals with chronic aphasia and controls disappeared after controlling for non-linguistic processing speed, reinforcing the notion that slowed processing may be a general consequence of stroke.

Although the number of impaired participants (per standard scores) did not differ between groups on the Flanker, PWA were significantly less accurate and slower than PWoA. PWA also exhibited poorer performance than PWoA on all examined DCCS Test measures. Unlike the PCPS Test, both the Flanker and DCCS Tests necessitate inhibition of non-target stimuli. The DCCS Test additionally requires shifting between two sets of rules (i.e., match per color or shape dimensions). These results suggest that compared to PWoA, PWA struggle with cognitive flexibility, particularly set-shifting. One caveat, however, is that executive control tests have been criticized for their inability to isolate specific cognitive domains (Miyake et al., 2000a). For example, the Wisconsin Card Sorting Task (WCST; Grant \& Berg, 1993)—another cognitive flexibility test-requires basic visual and numeric processing, abstract categorization, rule generation based on verbal feedback, and category/rule maintenance within (likely verbal) working memory (Miyake et al., 2000b). Unsurprisingly, given its potential language load, prior studies consistently report PWA exhibit worse performance on the WCST than controls and stroke survivors without aphasia (e.g., Baldo et al., 2015; Lee \& Pyun, 2014; Purdy, 2002). Unlike the WCST, explicit language demands of the Flanker and DCCS Test are minimal, and language impairment alone likely does not fully explain between-group differences in performance (e.g., Fucetola et al., 2009; Lee \& Pyun, 2014).

Furthermore, no language network ROIs were implicated in the executive control LASSO models (Fig. 2). Similar to Lacey et al. (2017), we found that frankly-damaged regions implicated in executive control deficits did not overlap with those implicated in anomia (i.e., classic language network regions IFGtri, IFGorb, STG, and pMTG). These findings imply a potential extralinguistic origin of executive control deficits within the sample-possibly reduced inhibition or cognitive flexibility-although this conclusion should be treated with caution given the small sample size. Consistent with prior LSM studies (Alyahya et al., 2020; Lacey et al., 2017; Schumacher et al., 2019), poorer performance on the DCCS Test was associated with damage to DLPFC. IPS damage was linked to poorer performance on both the
Flanker and DCCS Tests, a finding that accords with the role of the DAN in general (Fox et al., 2006) and the IPS's role in orienting attention to relevant stimuli (Humphreys \& Lambon Ralph, 2015).

Unlike ROI measures, similar hypoperfusion metrics were retained in the BNT and NIH Toolbox LASSO models, with parietal hypoperfusion retained across models. In acute stroke, hypoperfusion within vascular territories can result in severe impairments even when the infarct core is small (Beaulieu et al., 1999; Hillis et al., 2001, 2004, 2008). Because of this fact, including hypoperfusion measures within the LSM analyses was critical. Yet, a limitation of our approach was that the FHV hypoperfusion ratings lack voxel-level spatial sensitivity and cannot be localized to a specific network. Moreover, several variables were excluded from the LASSO models due to damage/hypoperfusion in too few participants. Excluded ROIs (see the Table 3 legend) were not restricted to one single network but were regions on the outskirts of the MCA territory. The ROI approach also lacked the whole-brain coverage and specificity of voxellevel methods used in other studies (e.g., Alyahya et al., 2020; Baldo et al., 2010; Lacey et al., 2017; Schumacher et al., 2019); additional regions or specific voxels within included ROIs may be identified in future studies via voxelwise LSM methods.

Another major study limitation was the small sample size, caused in large part by a pause in data collection for several months due to the COVID-19 pandemic. Additionally, while we analyzed age-corrected standard scores (which reflect comparison to a normative sample), we did not recruit additional healthy controls matched to patients across demographics. For all participants, testing was executed in their hospital room, and some distractions (e.g., sounds of medical equipment) could not be eliminated.

Despite these limitations, this study has important clinical implications. Our findings suggest high rates of executive dysfunction in acute LH stroke survivors: 12/13 PWA and 13/15 PWoA scored below normal limits per age-corrected standard scores on at least one NIH Toolbox measure. As such, executive control should - and can be-assessed in acute LH stroke survivors using the NIH Toolbox or similar measures. Given their impairments, patients may benefit from treatment directly targeting non-linguistic cognition (Peach, 2017), a notion not addressed in this work but necessary to investigate in future acute rehabilitation studies.

\section{Conclusions}

In all, these results indicate that acute LH stroke survivors with and without aphasia exhibit slowed processing and executive control deficits but that PWA particularly struggle 
with tasks requiring inhibition of non-target stimuli and setshifting. Lesion correlates of executive control deficits differed from naming impairments, suggesting a dissociation between lesion topography in behavioral profiles. Nonetheless, given the lack of significant differences between PWA and PWoA in most lesion metrics, future studies should include a larger acute stroke sample as well as measures of other brain variables (e.g., functional connectivity) that might additionally explain between-group differences in performance.

Supplementary Information The online version contains supplementary material available at https://doi.org/10.1007/s11682-021-00580-y.

Acknowledgements We gratefully acknowledge the individuals who participated in this study for their time and efforts spent. We thank Stroke Cognitive Outcomes and REcovery Lab members Alex Walker, Delaney Ubellacker, Colin Stein, Emilia Vitti, and Kristina Ruch for their assistance in data collection and Lisa Bunker for helping oversee operations of the larger project from which this study was generated.

Author Contributions Authorship contributions include study conception and design (ELM, CRK, and AEH), data collection (CRK and EBG), data processing (ELM, CRK, and EBG), statistical analysis (ELM), interpretation of the results (ELM and AEH), drafting the manuscript or revising it for important intellectual content (ELM, CRK, EBG, and AEH), and approving the final version to be published and agreeing to be accountable for the integrity and accuracy of all aspects of the work (ELM, CRK, EBG, and AEH).

Funding This study was funded by the NIH/NIDCD through grants R01DC05375 and P50 DC014664.

Data Availability The behavioral testing is reported in full within Table 2. Upon reasonable request, neuroimaging data and scripts can be made available to interested parties by contacting the first author.

\section{Declarations}

Ethical approval Study protocols were approved by the Johns Hopkins University School of Medicine Institutional Review Board in accordance with the Declaration of Helsinki.

Consent to participate Written informed consent was provided by each participant or in the case of individuals with impaired auditory and/or reading comprehension, by their healthcare proxy.

Consent to publish Not applicable.

Competing interests None of the authors have a conflict of interest to declare.

\section{References}

Alyahya, R. S. W., Halai, A. D., Conroy, P., \& Lambon Ralph, M. A. (2020). A unified model of post-stroke language deficits including discourse production and their neural correlates. Brain, 143(5), 1541-1554. https://doi.org/10.1093/brain/awaa074
Baldo, J., Dronkers, N., Wilkins, D., Ludy, C., Raskin, P., \& Kim, J. (2005). Is problem solving dependent on language? Brain and Language, 92(3), 240-250. https://doi.org/10.1016/j.bandl.2004. 06.103

Baldo, J. V., Bunge, S. A., Wilson, S. M., \& Dronkers, N. F. (2010). Is relational reasoning dependent on language? A voxel-based lesion symptom mapping study. Brain and Language, 113(2), 59-64. https://doi.org/10.1016/j.band1.2010.01.004

Baldo, J. V., Paulraj, S. R., Curran, B. C., \& Dronkers, N. F. (2015). Impaired reasoning and problem-solving in individuals with language impairment due to aphasia or language delay. Frontiers in Psychology, 6. https://doi.org/10.3389/fpsyg.2015.01523

Beaulieu, C., De Crespigny, A., Tong, D. C., Moseley, M. E., Albers, G. W., \& Marks, M. P. (1999). Longitudinal magnetic resonance imaging study of perfusion and diffusion in stroke: Evolution of lesion volume and correlation with clinical outcome. Annals of Neurology, 46(4), 568-578. https://doi.org/10.1002/15318249(199910)46:4\%3c568::AID-ANA4\%3e3.0.CO;2-R

Benjamini, Y., \& Hochberg, Y. (1995). Controlling the false discovery rate: A practical and powerful approach to multiple testing. 57(1), 289-300.

Bonini, M. V., \& Radanovic, M. (2015). Cognitive deficits in poststroke aphasia. Arquivos De Neuro-Psiquiatria, 73(10), 840-847. https://doi.org/10.1590/0004-282X20150133

Brett, M., Anton, J., Valabregue, R., \& Poline, J. B. (2002). Region of interest analysis using the MarsBar toolbox for SPM 99. 16(2), S497.

Butler, R. A., Lambon Ralph, M. A., \& Woollams, A. M. (2014). Capturing multidimensionality in stroke aphasia: Mapping principal behavioural components to neural structures. Brain, 137(12), 3248-3266. https://doi.org/10.1093/brain/awu286

Cole, M. W., Reynolds, J. R., Power, J. D., Repovs, G., Anticevic, A., \& Braver, T. S. (2013). Multi-task connectivity reveals flexible hubs for adaptive task control. Nature Neuroscience, 16(9), 1348-1355. https://doi.org/10.1038/nn.3470

El Hachioui, H., Visch-Brink, E. G., Lingsma, H. F., van de SandtKoenderman, M. W. M. E., Dippel, D. W. J., Koudstaal, P. J., \& Middelkoop, H. A. M. (2014). Non-linguistic cognitive impairment in poststroke aphasia: A prospective study. Neurorehabilitation and Neural Repair, 28(3), 273-281. https://doi.org/10.1177/ 1545968313508467

Engelter, S. T., Gostynski, M., Papa, S., Frei, M., Born, C., AjdacicGross, V., Gutzwiller, F., \& Lyrer, P. A. (2006). Epidemiology of aphasia attributable to first ischemic stroke: Incidence, severity, fluency, etiology, and thrombolysis. Stroke, 37(6), 1379-1384. https://doi.org/10.1161/01.STR.0000221815.64093.8c

Faroqi-Shah, Y., \& Gehman, M. (2021). The role of processing speed and cognitive control on word retrieval in aging and aphasia. Journal of Speech, Language, and Hearing Research, 64(3), 949-964. https://doi.org/10.1044/2020_JSLHR-20-00326

Fedorenko, E., \& Thompson-Schill, S. L. (2014). Reworking the language network. Trends in Cognitive Sciences, 18(3), 120-126. https://doi.org/10.1016/j.tics.2013.12.006

Fisher, N. J., Tierney, M. C., Snow, G. W., \& Szalai, J. P. (1999). Odd/ even short forms of the Boston naming test: Preliminary geriatric norms. The Clinical Neuropsychologist, 13(3), 359-364. https:// doi.org/10.1076/clin.13.3.359.1742

Flowers, H. L., Skoretz, S. A., Silver, F. L., Rochon, E., Fang, J., Flamand-Roze, C., \& Martino, R. (2016). Poststroke aphasia frequency, recovery, and outcomes: A systematic review and meta-analysis. Archives of Physical Medicine and Rehabilitation, 97(12), 2188-2201.e8. https://doi.org/10.1016/j.apmr.2016.03.006

Fonseca, J., Ferreira, J. J., \& Pavão Martins, I. (2017). Cognitive performance in aphasia due to stroke: A systematic review. International Journal on Disability and Human Development, 16(2). https://doi.org/10.1515/ijdhd-2016-0011 
Fox, M. D., Corbetta, M., Snyder, A. Z., Vincent, J. L., \& Raichle, M. E. (2006). Spontaneous neuronal activity distinguishes human dorsal and ventral attention systems. Proceedings of the National Academy of Sciences, 103(26), 10046-10051. https://doi.org/10. 1073/pnas.0604187103

Fridriksson, J., den Ouden, D.-B., Hillis, A. E., Hickok, G., Rorden, C., Basilakos, A., Yourganov, G., \& Bonilha, L. (2018). Anatomy of aphasia revisited. Brain, 141(3), 848-862. https://doi.org/10. 1093/brain/awx363

Friedman, J., Hastie, T., \& Tibshirani, R. (2010). Regularization paths for generalized linear models via coordinate descent. Journal of Statistical Software, 33(1), 1-22.

Fucetola, R., Connor, L. T., Strube, M. J., \& Corbetta, M. (2009). Unravelling nonverbal cognitive performance in acquired aphasia. Aphasiology, 23(12), 1418-1426. https://doi.org/10.1080/02687 030802514938

Gershon, R. C., Wagster, M. V., Hendrie, H. C., Fox, N. A., Cook, K. F., \& Nowinski, C. J. (2013). NIH Toolbox for assessment of neurological and behavioral function. Neurology, 80(Issue 11, Supplement 3), S2-S6. https://doi.org/10.1212/WNL.0b013e3182 $872 \mathrm{e} 5 \mathrm{f}$

Goldberg, E. B., Meier, E. L., Sheppard, S. M., Breining, B. L., \& Hillis, A. E. (2021). Stroke recurrence and its relationship with language abilities. Journal of Speech, Language, and Hearing Research, 1-16. https://doi.org/10.1044/2021_JSLHR-20-00347

Grant, D. A., \& Berg, E. A. (1993). Wisconsin Card Sorting Test. Psychological Assessment Resources.

Halai, A. D., Woollams, A. M., \& Lambon Ralph, M. A. (2017). Using principal component analysis to capture individual differences within a unified neuropsychological model of chronic post-stroke aphasia: Revealing the unique neural correlates of speech fluency, phonology and semantics. Cortex, 86, 275-289. https://doi.org/ 10.1016/j.cortex.2016.04.016

Halai, A. D., Woollams, A. M., \& Lambon Ralph, M. A. (2018). Triangulation of language-cognitive impairments, naming errors and their neural bases post-stroke. NeuroImage: Clinical, 17, 465-473. https://doi.org/10.1016/j.nicl.2017.10.037

Hartwigsen, G., \& Saur, D. (2019). Neuroimaging of stroke recovery from aphasia - Insights into plasticity of the human language network. NeuroImage, 190, 14-31. https://doi.org/10.1016/j.neuro image.2017.11.056

Heaton, R. K., Akshoomoff, N., Tulsky, D., Mungas, D., Weintraub, S., Dikmen, S., Beaumont, J., Casaletto, K. B., Conway, K., Slotkin, J., \& Gershon, R. (2014). Reliability and validity of composite scores from the NIH Toolbox Cognition Battery in adults. Journal of the International Neuropsychological Society, 20(6), 588-598. https://doi.org/10.1017/S1355617714000241

Hillis, A. E. (2007). Aphasia: Progress in the last quarter of a century. Neurology, 69(2), 200-213. https://doi.org/10.1212/01.wnl.00002 65600.69385.6f

Hillis, A. E., Barker, P. B., Wityk, R. J., Aldrich, E. M., Restrepo, L., Breese, E. L., \& Work, M. (2004). Variability in subcortical aphasia is due to variable sites of cortical hypoperfusion. Brain and Language, 89(3), 524-530. https://doi.org/10.1016/j.bandl. 2004.01.007

Hillis, A. E., Gold, L., Kannan, V., Cloutman, L., Kleinman, J. T., Newhart, M., Heidler-Gary, J., Davis, C., Aldrich, E., Llinas, R., \& Gottesman, R. F. (2008). Site of the ischemic penumbra as a predictor of potential for recovery of functions. Neurology, 71(3), 184-189. https://doi.org/10.1212/01.wnl.0000317091.17339.98

Hillis, A. E., Wityk, R. J., Tuffiash, E., Beauchamp, N. J., Jacobs, M. A., Barker, P. B., \& Selnes, O. A. (2001). Hypoperfusion of Wernicke's area predicts severity of semantic deficit in acute stroke. Annals of Neurology, 50(5), 561-566. https://doi.org/10.1002/ ana.1265
Humphreys, G. F., \& Lambon Ralph, M. A. (2015). Fusion and fission of cognitive functions in the human parietal cortex. Cerebral Cortex, 25(10), 3547-3560. https://doi.org/10.1093/cercor/bhu198

Keator, L. M., Faria, A. V., Kim, K. T., Saxena, S., Wright, A. E., Sheppard, S. M., Breining, B. L., Goldberg, E., Tippett, D. C., Meier, E., \& Hillis, A. E. (2020). An efficient bedside measure yields prognostic implications for language recovery in acute stroke patients. Cognitive and Behavioral Neurology, 33(3), 192-200. https://doi.org/10.1097/WNN.0000000000000238

Keil, K., \& Kaszniak, A. W. (2002). Examining executive function in individuals with brain injury: A review. Aphasiology, 16(3), 305-335. https://doi.org/10.1080/02687030143000654

Kertesz, A. (2007). Western aphasia battery: Revised. Pearson.

Keser, Z., Meier, E. L., Stockbridge, M. D., Breining, B. L., Sebastian, R., \& Hillis, A. E. (2021). Thalamic nuclei and thalamocortical pathways after left hemispheric stroke and their association with picture naming. Brain Connectivity, brain.2020.0831. https://doi. org/10.1089/brain.2020.0831

Keser, Z., Meier, E. L., Stockbridge, M. D., \& Hillis, A. E. (2020). The role of microstructural integrity of major language pathways in narrative speech in the first year after stroke. Journal of Stroke and Cerebrovascular Diseases, 29(9), 105078. https://doi.org/10. 1016/j.jstrokecerebrovasdis.2020.105078

Kiran, S. (2012). What Is the nature of poststroke language recovery and reorganization? ISRN Neurology, 2012, 1-13. https://doi.org/ $10.5402 / 2012 / 786872$

Korda, R. J., \& Douglas, J. M. (1997). Attention deficits in stroke patients with aphasia. Journal of Clinical and Experimental Neuropsychology, 19(4), 525-542. https://doi.org/10.1080/01688 639708403742

Kuzmina, E., \& Weekes, B. S. (2017). Role of cognitive control in language deficits in different types of aphasia. Aphasiology, 31(7), 765-792. https://doi.org/10.1080/02687038.2016.1263383

Lacey, E. H., Skipper-Kallal, L. M., Xing, S., Fama, M. E., \& Turkeltaub, P. E. (2017). Mapping common aphasia assessments to underlying cognitive processes and their neural substrates. Neurorehabilitation and Neural Repair, 31(5), 442-450.

Lee, B., \& Pyun, S.-B. (2014). Characteristics of cognitive impairment in patients with post-stroke aphasia. Annals of Rehabilitation Medicine, 38(6), 759. https://doi.org/10.5535/arm.2014.38.6.759

Lee, J. B., Kocherginsky, M., \& Cherney, L. R. (2020). Attention in individuals with aphasia: Performance on the Conners' Continuous Performance Test - 2nd edition. Neuropsychological Rehabilitation, 30(2), 249-265. https://doi.org/10.1080/09602011.2018. 1460852

Meier, E. L., Sheppard, S. M., Goldberg, E. B., Head, C. R., Ubellacker, D. M., Walker, A., \& Hillis, A. E. (2020). Naming errors and dysfunctional tissue metrics predict language recovery after acute left hemisphere stroke. Neuropsychologia, 148, 107651. https://doi.org/10.1016/j.neuropsychologia.2020.107651

Meinshausen, N., \& Yu, B. (2009). Lasso-type recovery of sparse representations for high-dimensional data. The Annals of Statistics, 37(1), 246-270. https://doi.org/10.1214/07-AOS582

Miyake, A., Emerson, M. J., \& Friedman, N. P. (2000). Assessment of executive functions in clinical settings: Problems and recommendations. Seminars in Speech and Language, Volume 21(Number 02), 0169-0183. https://doi.org/10.1055/s-2000-7563

Miyake, A., Friedman, N. P., Emerson, M. J., Witzki, A. H., Howerter, A., \& Wager, T. D. (2000b). The unity and diversity of executive functions and their contributions to complex "frontal lobe" tasks: A latent variable analysis. Cognitive Psychology, 41(1), 49-100. https://doi.org/10.1006/cogp.1999.0734

Nys, G. M. S., van Zandvoort, M. J. E., de Kort, P. L. M., van der Worp, H. B., Jansen, B. P. W., Algra, A., de Haan, E. H. F., \& Kappelle, L. J. (2005). The prognostic value of domain-specific cognitive 
abilities in acute first-ever stroke. Neurology, 64(5), 821-827. https://doi.org/10.1212/01.WNL.0000152984.28420.5A

Park, Y. H., Jang, J.-W., Park, S. Y., Wang, M. J., Lim, J.-S., Baek, M. J., Kim, B. J., Han, M.-K., Bae, H.-J., Ahn, S., \& Kim, S. (2015). Executive function as a strong predictor of recovery from disability in patients with acute stroke: A preliminary study. Journal of Stroke and Cerebrovascular Diseases, 24(3), 554-561. https://doi. org/10.1016/j.jstrokecerebrovasdis.2014.09.033

Peach, R. K. (2017). Cognitive approaches to aphasia treatment: Application of the cognition of language to aphasia intervention. Seminars in Speech and Language, 38(1), 3-4. https://doi.org/10. 1055/s-0036-1597259

Price, C. J. (2012). A review and synthesis of the first 20years of PET and fMRI studies of heard speech, spoken language and reading. NeuroImage, 62(2), 816-847. https://doi.org/10.1016/j.neuro image.2012.04.062

Purdy, M. (2002). Executive function ability in persons with aphasia. Aphasiology, 16(4-6), 549-557. https://doi.org/10.1080/02687 030244000176

Raichle, M. E., MacLeod, A. M., Snyder, A. Z., Powers, W. J., Gusnard, D. A., \& Shulman, G. L. (2001). A default mode of brain function. Proceedings of the National Academy of Sciences, 98(2), 676-682.

Reyes, D., Hitomi, E., Simpkins, A., Lynch, J., Hsia, A., Benson, R., Nadareishvili, Z., Luby, M., Latour, L., \& Leigh, R. (2017). Detection of perfusion deficits using FLAIR and GRE based vessel signs. Stroke, 48(suppl_1), ATP63-ATP63. https://doi.org/10. 1161/str.48.suppl_1.tp63

Rorden, C., Bonilha, L., Fridriksson, J., Bender, B., \& Karnath, H.-O. (2012). Age-specific CT and MRI templates for spatial normalization. NeuroImage, 61(4), 957-965. https://doi.org/10.1016/j.neuro image.2012.03.020

Schumacher, R., Halai, A. D., \& Lambon Ralph, M. A. (2019). Assessing and mapping language, attention and executive multidimensional deficits in stroke aphasia. Brain, 142(10), 3202-3216. https://doi.org/10.1093/brain/awz258

Simic, T., Rochon, E., Greco, E., \& Martino, R. (2019). Baseline executive control ability and its relationship to language therapy improvements in post-stroke aphasia: A systematic review. Neuropsychological Rehabilitation, 29(3), 395-439. https://doi.org/ 10.1080/09602011.2017.1307768

Spaccavento, S., Marinelli, C. V., Nardulli, R., Macchitella, L., Bivona, U., Piccardi, L., Zoccolotti, P., \& Angelelli, P. (2019). Attention deficits in stroke patients: The role of lesion characteristics, time from stroke, and concomitant neuropsychological deficits. Behavioural Neurology, 2019, 1-12. https://doi.org/10.1155/2019/78357 10

Tulsky, D. S., Holdnack, J. A., Cohen, M. L., Heaton, R. K., Carlozzi, N. E., Wong, A. W. K., Boulton, A. J., \& Heinemann, A. W. (2017). Factor structure of the NIH Toolbox Cognition Battery in individuals with acquired brain injury. Rehabilitation Psychology, 62(4), 435-442. https://doi.org/10.1037/rep0000183

Villard, S., \& Kiran, S. (2015). Between-session intra-individual variability in sustained, selective, and integrational non-linguistic attention in aphasia. Neuropsychologia, 66, 204-212. https://doi. org/10.1016/j.neuropsychologia.2014.11.026

Villard, S., \& Kiran, S. (2018). Between-session and within-session intra-individual variability in attention in aphasia. Neuropsychologia, 109, 95-106. https://doi.org/10.1016/j.neuropsychologia. 2017.12.005

Weintraub, S., Dikmen, S. S., Heaton, R. K., Tulsky, D. S., Zelazo, P. D., Slotkin, J., Carlozzi, N. E., Bauer, P. J., Wallner-Allen, K., Fox, N., Havlik, R., Beaumont, J. L., Mungas, D., Manly, J. J., Moy, C., Conway, K., Edwards, E., Nowinski, C. J., \& Gershon, R. (2014). The Cognition Battery of the NIH Toolbox for assessment of neurological and behavioral function: Validation in an adult sample. Journal of the International Neuropsychological Society, 20(6), 567-578. https://doi.org/10.1017/S1355617714000320

Yao, J., Liu, X., Liu, Q., Wang, J., Ye, N., Lu, X., Zhao, Y., Chen, H., Han, Z., Yu, M., Wang, Y., Liu, G., \& Zhang, Y. (2020). Characteristics of non-linguistic cognitive impairment in post-stroke aphasia patients. Frontiers in Neurology, 11, 1038. https://doi.org/ 10.3389/fneur.2020.01038

Zinn, S., Bosworth, H. B., Hoenig, H. M., \& Swartzwelder, H. S. (2007). Executive function deficits in acute stroke. Archives of Physical Medicine and Rehabilitation, 88(2), 173-180. https:// doi.org/10.1016/j.apmr.2006.11.015

Publisher's Note Springer Nature remains neutral with regard to jurisdictional claims in published maps and institutional affiliations. 